Asian J Agric \& Biol. 2020;8(2):129-137.

\title{
Weed control in wheat through different sorghum formulations as an organic herbicide
}

\author{
Muhammad Waqas Aslam Cheema ${ }^{1,2}$, Tassadduq Rasool1,3*, Hassan Munir ${ }^{1}$, Muhammad Mazhar Iqbal', \\ Tayyaba Naz', Muhammad Ikram-ul-Haq ${ }^{1}$, Ahmad Mustafa ${ }^{1}$, Mubashar Nadeem ${ }^{1}$, Sami Ullah ${ }^{6}$ \\ ${ }^{1}$ Department of Agronomy, University of Agriculture, Faisalabad, Pakistan \\ ${ }^{2}$ Pakistan Oilseed Board Development, Ministry of National Food Security and Research, Islamabad, Pakistan \\ ${ }^{3}$ Directorate General Soil Survey, Punjab, Lahore, Pakistan \\ ${ }^{4}$ Soil and Water Testing Laboratory, Chiniot, Ayub Agricultural Research Institute, Faisalabad, Pakistan \\ ${ }^{5}$ Institute of Soil and Environmental Sciences, University of Agriculture, Faisalabad, Pakistan \\ ${ }^{6}$ PARC Arid Zone Research Institute, D.I. Khan, Pakistan
}

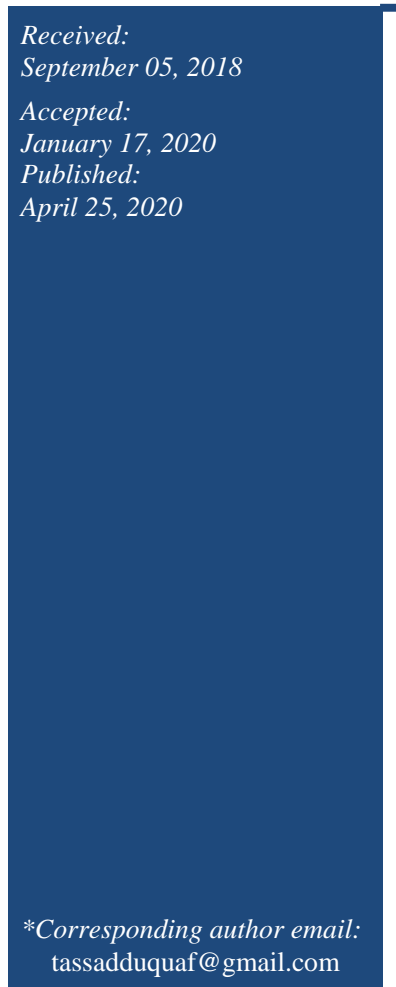

\begin{abstract}
The impact of phytotoxic potential of sorghum formations and herbicide application along with their comparative economic advantages over each other in the wheat crop, was evaluated in present filed study. The sorghum formulations with their application volumes were as i) the water soaked extract $\left(330 \mathrm{~L} \mathrm{ha}^{-1}\right)$, ii) water boiled extract (15, 20,25 or $\left.30 \mathrm{~L} \mathrm{ha}^{-1}\right)$, iii) fine powdered $\left(270,360,450\right.$ or $\left.540 \mathrm{~g} \mathrm{ha}^{-1}\right)$. Most of the sorghum formulations controlled weed density in array $24-61 \%$. The weeds dry weight was declined by $20-59 \%$ and crop yield raised upto $25 \%$. The water soaked extract at a volume of $330 \mathrm{~L} \mathrm{ha}^{-1}$ and water boiled extract at $20 \mathrm{~L} \mathrm{ha}^{-1}$ volume were not only useful in weed control but also enhanced yield and demonstrated economic profitability. These treatments provided higher rates of marginal returns \% $154.54 \$$ and $47.70 \$$, respectively. Although, sorghum powder were effective in weed control but were uneconomical because of their high production cost.
\end{abstract}

Keywords: Allelopathy, Toxins, Weeds, Wheat, Herbicides

\section{How to cite this:}

Cheema MWA, Rasool T, Munir H, Iqbal MM, Naz T, Haq MIU, Mustafa A, Nadeem $\mathrm{M}$ and Ullah S, 2020. Weed control in wheat through different sorghum formulations as an organic herbicide. Asian J. Agric. Biol. 8(2):129-137. DOI: https://doi.org/10.35495/ajab.2018.09.285

This is an Open Access article distributed under the terms of the Creative Commons Attribution 3.0 License. (https://creativecommons.org/licenses/by/3.0), which permits unrestricted use, distribution, and reproduction in any medium, provided the original work is properly cited.

\section{Introduction}

Weeds are the potential competing factor in the agroecosystem which can deprive the crops from necessary growth factors including space, water and nutrients (Bilalis et al., 2010). The concerns in changing environments need special consideration for weed management without chemical approach
(Corley, 2015; Kadioglu and Farooq, 2017; Glab et al., 2017). Irrespective of the resource competing factors, the crops are also subjected to the toxic allelochemicals of weeds released by exudation into the rhizosphere and can inhibit germination, growth and yield (Zohaib et al., 2014). The estimated wheat yield losses owing to establishment of weeds are up to $25-30 \%$. There are various approaches for the 
management of weeds such as chemical, mechanical, bio-herbicidal and agronomic management practices (Cheema and Khaliq, 2000; Bilalis et al., 2010). From the above reports, chemical weed control through herbicides is considered a resourceful weed management method (Torun and Uygur, 2012). However, application of herbicides has posed serious threats to the environment and raised health concerns (Zhu and Li, 2002; Khan and Damlas, 2015; Corley, 2015). Certain other problems are also arising because of induced herbicide resistance even against herbicides such as glyphosate. Organic agriculture focuses on the sustainable solution to weed and insect problem through bio-herbicides (Cheema et al., 2004; Smith et al., 2015; Silva et al., 2015). Bio-herbicides are chemicals which originate from the internal metabolism of plants and are entered into ecosystem via leaching process, exudation of roots, volatilized from live plants parts, or from decomposition of residues. Allelopathy phenomenon is due to the allelochemicals which contain various chemicals group types. The prominent chemical groups are cummins, flavonoids, alkaloids and terpenes (Mazid et al., 2011). The most commonly observed effects are on growth of seedling and cell meristematic activity (Glab et al., 2017). Sorgoleone (sorghum most studied allelochemical) blocks ATP synthesis, while benzoic acid interrupts essential element absorption in plants, blocks photosystem-II, carbon assimilation and photochromic activity (Mahmood and Cheema, 2004; Farooq et al., 2011, 2013).

Sorghum is established an allelopathic plant, which contain several chemicals of different nature (Anne et al., 2009; Farooq et al., 2013; Weston et al., 2013). These are identified and named as sorgoleone, vanillic acid, syringic acid, ferulic acid, p-hydroxy-benzoic acid, gallic acid, phenols, p-coumaric acid and 3deoxy anthocyanidin derivatives (Weston et al., 2013). Sorgoleone has been recognized to influence the electron transport chain in photosystem-I and photosystem-II, thus giving it a bio-herbicidal recognition (Glab et al., 2017). As far as the application of sorghum allelopathy is concerned, its field appraisal needs to be evaluated under the given circumstances. Therefore, this research was intended to assess the phytotoxic potential of sorghum by making water extract and dried powder in comparison with herbicide.

\section{Material and Methods}

\section{Climate of the study site and characteristics of used soil}

The study was conceded at Agronomic Research Area, $\left(31^{\circ} 27^{\prime} \mathrm{N}, 73^{\circ} 08^{\prime} \mathrm{E}, 184\right.$ meters above sea level), University of Agriculture, Faisalabad, Pakistan. The constituency has a semi-arid climate along with very hot summer and cool up to $2{ }^{\circ} \mathrm{C}$ in January. Wheat growing season spans from October to the first quarter of May.

Soil samples were analyzed for physico-chemical properties including texture, saturation paste $\mathrm{pH}$, soil organic matter (SOM), available phosphorus and potassium following Iqbal et al. (2017). The obtained data are presented in Table 1.

Table-1: Physico-chemical properties of soil used for experiment and meteorological data

\begin{tabular}{|l|c|}
\hline \multicolumn{1}{|c|}{ Parameter } & Value \\
\hline Soil type & Clay Loam \\
\hline Sand (\%) & 20 \\
\hline Silt (\%) & 45 \\
\hline Clay (\%) & 35 \\
\hline $\mathrm{pH}$ & 7.5 \\
\hline Soil Organic Matter $(\%)$ & 0.97 \\
\hline Total Nitrogen (\%) & 0.10 \\
\hline Available Phosphorus $\left(\mathrm{mg} \mathrm{kg}^{-1}\right)$ & 15.2 \\
\hline Available Potassium $\left(\mathrm{mg} \mathrm{kg}{ }^{-1}\right)$ & 240 \\
\hline Meteorological data & $(2011-12)$ \\
\hline $\begin{array}{l}\text { Climate }(\text { Köppen-Geiger } \\
\text { classification) }\end{array}$ & $\begin{array}{c}\text { Semi-arid (very hot and } \\
\text { humid summers and dry } \\
\text { cool winters. })\end{array}$ \\
\hline Annual ave. Temperature $\left({ }^{\circ} \mathrm{C}\right)$ & \\
\hline Ave. Highest Temp. $\left({ }^{\circ} \mathrm{C}\right)$ & 31.6 \\
\hline Ave. Lowest Temp. $\left({ }^{\circ} \mathrm{C}\right)$ & 17.4 \\
\hline Total precipitation $(\mathrm{mm})$ & 346 \\
\hline
\end{tabular}

\section{Sorghum formulations}

The following treatments were planned with a concentration on per hectare basis viz, a weedy check (control), herbicide application, water-soaked, boiled and powdered sorghum formulations in various concentrations. Boiled sorghum extract solutions were made with concentrations @ 15, 20,25 or $30 \mathrm{~L} \mathrm{ha}^{-1}$, which were further added into 100 liters water containers as to make the volume upto enough level for spray over the one hectare. Likewise, the powder formulations were 270, 360, 450 and $540 \mathrm{~g} \mathrm{ha}^{-1}$. Moreover, sorghum water soaked extract $330 \mathrm{~L} \mathrm{ha}^{-1}$ 
and pre-emergence herbicide iodosulforun + mesosulforun (Atlantis 3.6 WG) $14.4 \mathrm{~g} \mathrm{ha}^{-1}$ a.i. were also included in this study.

Sorghum water extract was geared up in allelopathy laboratory subsequent the procedures adopted by Cheema and Khaliq (2000). In procedure, mature sorghum crop (cultivar JS-263) was used for all these treatment formulations. Sorghum above ground parts were harvested and placed in the shade for a couple of days to avoid leaching by rain. After drying in the shade, it was chopped by ordinary fodder cutter. Thereafter, it was used for water-soaked, boiled water extraction, and powder formulations. The small cut pieces of sorghum were immersed in $1: 10$ ratio $(1 \mathrm{~kg}$ in 10 liters) for 24 hours and concentrated to reduce its volume by $95 \%$ via boiling at $212^{\circ} \mathrm{F}$ for boiled water extractions. Sorghum powder was prepared through a spray drier and prepared through hot evaporation method. One liter of sorghum extract yielded about $18 \mathrm{~g}$ of sorghum powder. The calibrated volume of spray was equivalent to $330 \mathrm{~L} \mathrm{ha}^{-1}$. The application of formulations was made by means of Knapsack sprayer with T-jet nozzle. Sorghum application was made 30 days after sowing and after the emergence of wheat. At $30 \mathrm{DAS}$, wheat was at the stage of tillering (GS-29 i.e., growing stage 29 with 59 tillers; Phalaris minor: 3-4 leaf stage, Avena fatua; 3 leaf stage).

\section{Crop management practices}

The experiment was divided into six blocks and planned as randomized complete block design. Wheat was planted in $25 \mathrm{~cm}$ apart rows in plot dimensions $(2 \mathrm{~m} \times 7 \mathrm{~m})$ with 8 rows in each plot. Planting was made in uniformly-prepared soil with seeding rate $125 \mathrm{~kg}$ $\mathrm{ha}^{-1}$. Fertilization was done at $100-90-75 \mathrm{~kg} \mathrm{ha}^{-1}$ as $\mathrm{N}: \mathrm{P}: \mathrm{K}$ half on soil formulations and remain in two slits. Irrigation was applied at stage 2 (tiller beginning), stage 7 (formation of secondary node of stem), stage 10.4 (three-quarter head emerged) and stage 11.1 (milky ripe stage) according to the Feekes scale of cereal crops (Large, 1954).

\section{Data collection and interpretation}

Weed density measurement was made by using a quadrate $(1 \mathrm{~m} \times 1 \mathrm{~m})$ dimension at 3 places after 35 and 60 days of sowing. Weeds (Phalaris minor and Chenopodium album) were also removed for dry weight measurement from the same plots. The yield and other related traits were measured at maturity. Harvesting was done manually and threshed with a small mechanical thresher. The data presented is the average of various recorded observations. The weeds dry weight was determined by oven-drying at $75^{\circ} \mathrm{C}$ for the period of 48 hours and weighed till constant observation. Data interpretation was made by Fisher's analysis of variance technique using Statistix 8.2 and treatment differences were estimated employing the LSD test at $5 \%$ probability. Data normally distributed and used without any transformation to execute ANOVA.

\section{Economic analysis}

Economic efficiency of various treatments was evaluated according to the method devised by Byerlee (1988). According to this method, the fixed cost (nontreatment) comprise seed, fertilizer and machinery inputs and variable cost (treatment expenditure). A $10 \%$ yield reduction (Adjusted yield) is made for economic analysis, taking into consideration the loss that occurs due to harvesting and transport from field to market by the farmers (Arif et al., 2015). Net benefits were obtained by subtracting every variable cost (i.e. herbicide, cost of formulations of water soaked extractions, sorghum allelopathic powder, rent of sprayer machine or spray labour cost) as of gross income. The calculations are in local currency (Pak rupee) for estimation of various costs and to make easy calculations ( 1 Dollar $=104.82$ Rs. $)$.

\section{Marginal rate of returns (MRR\%)}

The marginal analysis determines the dominance of a treatment on the preceding treatment and estimated through ordering the treatments in increasing direction and the MRR was calculated using following formula:

$$
\mathrm{MRR} \%=\frac{\text { marginal net benefits }(\text { Rs. })}{\text { marginal expenditures }(\text { Rs. })} \times 100
$$

\section{Results}

Weed flora at the investigational place mainly include two weed species i.e., little seed canary grass (Phalaris minor) and lambquarters (Chenopodium album). 
Muhammad Waqas Aslam Cheema et al.

Table-2: Effect of sorghum water extract, sorghum powder formation, and sorghum soaked water extract on weed density

\begin{tabular}{|c|c|c|c|c|c|c|}
\hline Treatments & $\begin{array}{l}\text { Weed density } \\
\text { (30 DAS) }\end{array}$ & $\begin{array}{l}\text { Weed density } \\
\text { (60 DAS) }\end{array}$ & $\begin{array}{c}\text { Density of } P . \\
\text { minor } \\
\text { (30 DAS })\end{array}$ & $\begin{array}{c}\text { Density of } P . \\
\text { minor } \\
(60 \text { DAS })\end{array}$ & $\begin{array}{l}\text { Density of } C . \\
\text { album (30 DAS) }\end{array}$ & $\begin{array}{l}\text { Density of } C \text {. } \\
\text { album (60 DAS) }\end{array}$ \\
\hline Weedy check & $36.2 \mathrm{a}$ & $49.0 \mathrm{a}$ & $19.7 \mathrm{ab}$ & $17.0 \mathrm{a}$ & $16.5 \mathrm{a}$ & $9.2 \mathrm{a}$ \\
\hline Water application (25 DAS) & $33.2 \mathrm{ab}(9 \%)$ & $48.7 \mathrm{a}(1 \%)$ & $21.5 \mathrm{a}(13 \%)$ & $13.0 \mathrm{abc}(23 \%)$ & $11.7 \mathrm{ab}(28.78 \%)$ & $4.2 \mathrm{ab}(54.04 \%)$ \\
\hline Herbicide application* (25 DAS) & $9.5 \mathrm{e}(73 \%)$ & $18.0 \mathrm{e}(64 \%)$ & $5.0 \mathrm{~d}(75 \%)$ & $3.7 \mathrm{~d}(78 \%)$ & $4.5 \mathrm{c}(72.72 \%)$ & $2.7 \mathrm{~b}(70.27 \%)$ \\
\hline $\begin{array}{l}\text { Sorghum water soaked extract solution } \\
@ 330 \mathrm{~L} \mathrm{ha}^{-1}(25 \mathrm{DAS})\end{array}$ & $25.7 \mathrm{abcd}(28 \%)$ & $27.5 \mathrm{cde}(43 \%)$ & $16.2 \mathrm{abc}(18 \%)$ & $7.2 \mathrm{~cd}(57 \%)$ & 9.5 bc $(42.42 \%)$ & $3.7 \mathrm{ab}(59.45 \%)$ \\
\hline $\begin{array}{l}\text { Sorghum boiled water extract } \\
\text { @ } 15 \mathrm{~L} \mathrm{ha}^{-1}(25 \mathrm{DAS})\end{array}$ & $27.5 \mathrm{abc}(24 \%)$ & $44.5 \mathrm{ab}(9 \%)$ & $14.2 \mathrm{abc}(27 \%)$ & $14.2 \mathrm{ab}(16 \%)$ & $13.2 \mathrm{ab}(19.69 \%)$ & $3.7 \mathrm{ab}(59.45 \%)$ \\
\hline $\begin{array}{l}\text { Sorghum boiled water extract @ } 20 \mathrm{~L} \mathrm{ha}^{-1} \\
\text { (25 DAS) }\end{array}$ & 16.7 de $(53 \%)$ & $31.2 \mathrm{~cd}(34 \%)$ & $9.0 \mathrm{~cd}(56 \%)$ & $7.7 \mathrm{~cd}(53 \%)$ & 7.7 bc $(53.03 \%)$ & $4.2 \mathrm{ab}(54.05 \%)$ \\
\hline $\begin{array}{l}\text { Sorghum boiled water extract } 25 \mathrm{~L} \mathrm{ha}^{-1} \\
\text { (@ 25 DAS) }\end{array}$ & $26.7 \mathrm{abcd}(26 \%)$ & 20.7 de $(57 \%)$ & 16.0 abc $(19 \%)$ & $8.7 \mathrm{~d}(51 \%)$ & $10.7 \mathrm{abc}(34.48 \%)$ & $2.7 \mathrm{~b}(70.27 \%)$ \\
\hline $\begin{array}{l}\text { Sorghum boiled water extract @ } 30 \mathrm{~L} \mathrm{ha}^{-1} \\
\text { (25 DAS) }\end{array}$ & $22.2 \mathrm{~cd}(38.76 \%)$ & $36.0 \mathrm{bc}(26 \%)$ & $13.7 \mathrm{abc}(31 \%)$ & 11.0abcd $(35 \%)$ & $8.5 \mathrm{bc}(48.48 \%)$ & $7.0 \mathrm{ab}(24.32 \%)$ \\
\hline Sorghum powder @ 270 g ha ${ }^{-1}$ (25 DAS) & 25.2 bcd $(38.7 \%)$ & $21.2 \mathrm{de}(58 \%)$ & $14.5 \mathrm{abc}(26 \%)$ & $8.0 \mathrm{~cd}(53 \%)$ & $10.7 \mathrm{abc}(34.48 \%)$ & $3.0 \mathrm{~b}(67.56 \%)$ \\
\hline Sorghum powder @ $360 \mathrm{~g} \mathrm{ha}^{-1}$ (25 DAS) & $21.5 \mathrm{~cd}(41 \%)$ & 26.7 cde $(45 \%)$ & $13.5 \mathrm{bc}(31 \%)$ & 9.0 bcd $(47 \%)$ & $8.0 \mathrm{bc}(51.51 \%)$ & $4.0 \mathrm{ab}(56.75 \%)$ \\
\hline Sorghum powder@450 g ha-1 (25 DAS) & 24.5 bcd $(32 \%)$ & $34.5 \mathrm{bc}(30 \%)$ & $15.0 \mathrm{abc}(24 \%)$ & $13.2 \mathrm{abc}(22)$ & $9.5 \mathrm{bc}(42.42 \%)$ & $7.2 \mathrm{ab}(21.62 \%)$ \\
\hline Sorghum powder@ $@ 540 \mathrm{~g} \mathrm{ha}^{-1}(25 \mathrm{DAS})$ & 30.5 abc $(16 \%)$ & $22.5 \mathrm{de}(56 \%)$ & $17.0 \mathrm{ab}(13 \%)$ & $8.5 \mathrm{~d}(50 \%)$ & $13.5 \mathrm{ab}(18.18 \%)$ & $3.7 \mathrm{ab}(59.45 \%)$ \\
\hline LSD value $\mathrm{p}<0.05$ & 10.6 & 6.3 & 7.8 & 6.3 & 12.0 & 4.3 \\
\hline
\end{tabular}

\section{Weed density}

Weed density was considerably inclined by diverse sorghum formulations and applied herbicide. The maximum weed density was observed in control (weedy check). Whereas, herbicide application resulted in minimum values (Table 2).

Among the sorghum formulations, a significant weed reduction $(53 \%)$ in weed density was observed through the sorghum boiled water extract @ $20 \mathrm{~L} \mathrm{ha}^{-1}$ in comparison with control (weedy check) after 30 days of wheat sowing. While $25 \mathrm{~L}$ water boiled sorghum extract was effective (57\% reduction) 60 days after sowing. It was observed a considerable variation in weed control between different species. For example, the sorghum formulations controlled Phalaris minor by 20-57\% and Chenopodium album by 21-67\%. Irrespective of considerable reductions by sorghum formulations, herbicide reduced weed density by $73 \%$ and $64 \%$ after 30 and 60 DAS.

\section{Weeds dry weight}

Weeds DW determined the biomass reduction of weeds under study. Most of the treatments suppressed weeds, thereby reducing the DW (Table 3). It was observed that sorghum powder in an amount of $360 \mathrm{~g}$ $\mathrm{ha}^{-1}$ considerably declined the weeds DW by $59 \%$, and sorghum water extract at application of volume $20 \mathrm{~L}$ $\mathrm{ha}^{-1}$ was efficient to lessen the overall weeds dry weight by $61 \%$ than control. The decrease in the weeds DW by the boiled water extracts of sorghum (at volumes of 15, 20, 25 and $30 \mathrm{~L} \mathrm{ha}^{-1}$ ) extend from $35 \%$ to $61 \%$, estimated on 30 and 60 DAS. While sorghum powder weights viz. 270, 360, 450 and $540 \mathrm{~g} \mathrm{ha}^{-1}$, declined weed DW $24 \%$ to $59 \%$ at the respective observational durations. The application of water soaked extraction, declined weeds DW from 50 to $59 \%$ estimated at the same 30 and 60 DAS. Overall the highest reduction in DW was observed under herbicide application.

\section{Growth and yield-related attributes}

Highest growth and yield-related attributes were observed, when herbicide Atlantis (3.6 WG; iodosulforun + mesosulforun) at a concentration of $14.4 \mathrm{~g} \mathrm{ha}^{-1}$. For wheat, the biological and economic yields were increased significantly $(\mathrm{p} \leq 0.05)$ due to sorghum formulations application in the range of 9$25 \%$ than control.

\section{Economic analysis}

Economic analysis is presented in the Tables 5 and 6 and it was observed that sorghum treatments remained economically valuable. The highest net benefits 1056 $\$$ over control came from the sorghum boiled water treatment when it was applied at a volume of 20 liters per hectares. 
Table-3: Effect of application of various sorghum formulations on weeds dry weight present in wheat $\left(\mathrm{g} / \mathrm{m}^{2}\right)$

\begin{tabular}{|c|c|c|c|c|c|c|}
\hline Treatments & $\begin{array}{l}\text { Weeds dry } \\
\text { weight } \\
\text { (30 DAS) }\end{array}$ & $\begin{array}{l}\text { Weeds dry } \\
\text { weight } \\
\text { (60 DAS) }\end{array}$ & $\begin{array}{l}\text { Dry weight of } \\
\text { Phalaris } \\
\text { minor } \\
\text { (30 DAS) }\end{array}$ & $\begin{array}{c}\text { Dry weight of } \\
\text { Phalaris } \\
\text { minor } \\
(60 \text { DAS })\end{array}$ & $\begin{array}{c}\text { Dry weight of } \\
\text { Chenopodium } \\
\text { album } \\
\text { (30 DAS) }\end{array}$ & $\begin{array}{c}\text { Dry weight of } \\
\text { Chenopodium } \\
\text { album } \\
\text { (60 DAS })\end{array}$ \\
\hline Weedy check & $0.9 \mathrm{a}$ & $11.5 \mathrm{a}$ & $0.35 \mathrm{a}$ & $2.6 \mathrm{a}$ & $0.64 \mathrm{a}$ & $3.33 \mathrm{a}$ \\
\hline Water application (25 DAS) & $0.80 \mathrm{ab}(11 \%)$ & $9.74 \mathrm{a}(15 \%)$ & $0.30 \mathrm{a}(14 \%)$ & $2.3 \mathrm{ab}(12 \%)$ & $0.50 \mathrm{ab}(22 \%)$ & $2.81 \mathrm{ab}(15 \%)$ \\
\hline Herbicide application* (25 DAS) & 0.15 e $(83 \%)$ & $1.49 \mathrm{~d}(88 \%)$ & $0.07 \mathrm{c}(80 \%)$ & $0.40 \mathrm{~d}(84 \%)$ & 0.07 e $(90 \%)$ & 0.23 e $(93 \%)$ \\
\hline $\begin{array}{l}\text { Sorghum water soaked extract solution } \\
@ 330 \mathrm{~L} \mathrm{ha}^{-1}(25 \mathrm{DAS})\end{array}$ & $0.45 \mathrm{~cd}(50 \%)$ & $4.59 \mathrm{bcd}(59 \%)$ & $0.21 \mathrm{ab}(40 \%)$ & $1.04 \mathrm{~cd}(60 \%)$ & $0.30 \mathrm{~cd}(54 \%)$ & $1.54 \mathrm{~cd}(54 \%)$ \\
\hline Sorghum boiled water extract @ $15 \mathrm{~L} \mathrm{ha}^{-1}$ (25 DAS) & $0.59 \mathrm{bc}(35 \%)$ & $6.89 \mathrm{~b}(41 \%)$ & $0.16 \mathrm{bc}(54 \%)$ & $1.89 \mathrm{abc}(28 \%)$ & $0.40 \mathrm{bc}(38 \%)$ & 2.02 bc $(39 \%)$ \\
\hline Sorghum boiled water extract @ $20 \mathrm{~L} \mathrm{ha}^{-1}$ (25 DAS) & $0.41 \mathrm{~cd}(54 \%)$ & $4.56 \mathrm{bcd}(61 \%)$ & $0.14 \mathrm{bc}(59 \%)$ & $1.16 \mathrm{bcd}(56 \%)$ & 0.27 cde $(58 \%)$ & $1.43 \mathrm{~cd}(58 \%)$ \\
\hline Sorghum boiled water extract 25 L ha ${ }^{-1}$ (@ 25 DAS) & $0.47 \mathrm{~cd}(48 \%)$ & 4.62 bcd $(59 \%)$ & $0.18 \mathrm{bc}(50 \%)$ & 1.24 bcd $(52 \%)$ & 0.22 cde $(63 \%)$ & $1.37 \mathrm{~cd}(59 \%)$ \\
\hline Sorghum boiled water extract @ 30 L ha ${ }^{-1}$ (25 DAS) & $0.56 \mathrm{bc}(38 \%)$ & $6.52 \mathrm{~b}(44 \%)$ & $0.19 \mathrm{bc}(46 \%)$ & $2.11 \mathrm{abc}(19 \%)$ & $0.35 \mathrm{~cd}(46 \%)$ & $1.97 \mathrm{bc}(41 \%)$ \\
\hline Sorghum powder @ 270 $\mathrm{g} \mathrm{ha}^{-1}(25 \mathrm{DAS})$ & $0.59 \mathrm{bc}(34 \%)$ & 5.83 bc $(49 \%)$ & $0.27 \mathrm{ab}(23 \%)$ & $1.31 \mathrm{bcd}(50 \%)$ & $0.32 \mathrm{~cd}(50 \%)$ & $1.57 \mathrm{~cd}(53 \%)$ \\
\hline Sorghum powder @ $360 \mathrm{~g} \mathrm{ha}^{-1}$ (25 DAS) & $0.36(59 \%)$ de & $5.70 \mathrm{bc}(50 \%)$ & $0.23 \mathrm{ab}(35 \%)$ & 1.18 bcd $(55 \%)$ & 0.24 cde $(61 \%)$ & $1.46 \mathrm{~cd}(57 \%)$ \\
\hline Sorghum powder@ @ 450 $\mathrm{g} \mathrm{ha}^{-1}$ (25 DAS) & $0.38(58 \%) \mathrm{de}$ & $5.350 \mathrm{bc}(54 \%)$ & $0.21 \mathrm{bc}(40 \%)$ & 1.96 abc $(24 \%)$ & 0.25 cde $(60 \%)$ & $1.95 \mathrm{ab}(42 \%)$ \\
\hline Sorghum powder @ 540 $\mathrm{g} \mathrm{ha}^{-1}(25 \mathrm{DAS})$ & $0.69(24 \%) \mathrm{b}$ & 5.73 bc $(50 \%)$ & $0.32 \mathrm{~A}(13 \%)$ & 1.27 bcd $(51 \%)$ & $0.39 \mathrm{bc}(40 \%)$ & $1.52 \mathrm{~cd}(53 \%)$ \\
\hline LSD Value $\mathrm{p}<0.05$ & 0.24 & 2.63 & 0.23 & 1.23 & 0.17 & 1.1344 \\
\hline
\end{tabular}

Table-4: Effect of application of various sorghum formulations on growth and yield components of wheat

\begin{tabular}{|c|c|c|c|c|c|}
\hline Treatments & \begin{tabular}{|c|}
$\begin{array}{c}\text { Harvest index } \\
(\%)\end{array}$ \\
\end{tabular} & $\begin{array}{c}\text { Biological yield } \\
\left.\text { (tons ha }^{-1}\right)\end{array}$ & \begin{tabular}{|c|}
$\begin{array}{c}\text { Economic yield } \\
\left(\text { tons } \text { ha }^{-1}\right)\end{array}$ \\
\end{tabular} & $\begin{array}{l}\text { Plant height } \\
(\mathrm{cm})\end{array}$ & $\begin{array}{c}\text { Leaf area } \\
\left(\mathrm{cm}^{2}\right)\end{array}$ \\
\hline Weedy check & $33.7 \mathrm{e}$ & $9.3 \mathrm{f}$ & $3.5 \mathrm{f}$ & $87.3 \mathrm{~cd}$ & $178.9 \mathrm{~d}$ \\
\hline Water application (25 DAS) & $33.2 \mathrm{e}$ & $9.4 \mathrm{f}(10.6 \%)$ & 3.8 ef $(5.7 \%)$ & 88.7 bcd $(1.5 \%)$ & $212.8 \mathrm{c}$ \\
\hline $\begin{array}{c}\text { Herbicide application iodosulforun }+ \text { mesosulforun @ } 14.4 \mathrm{~g} \mathrm{ha}^{-1} \\
\text { (Atlantis @ 3.6WG }\end{array}$ & $47.5 \mathrm{a}$ & $12.2 \mathrm{a}(25 \%)$ & $5.0 \mathrm{a}(29.5 \%)$ & $98.5 \mathrm{a}(11.2 \%)$ & $267.2 \mathrm{a}$ \\
\hline Sorghum water soaked extract solution @ 330 L ha $^{-1}(25$ DAS) & $42.7 \mathrm{abcd}$ & $10.8 \mathrm{cde}(14.6 \%)$ & $4.6 \mathrm{abc}(22 \%)$ & $93.2 \mathrm{ab}(6.3 \%)$ & $251.7 \mathrm{a}$ \\
\hline Sorghum boiled water extract@15 L ha ${ }^{-1}(25$ DAS) & 37.2 cde & 10.1 ef $(8.0 \%)$ & $3.9 \operatorname{def}(10 \%)$ & $86.7 \mathrm{~d}(72 \%)$ & $224.4 \mathrm{bc}$ \\
\hline Sorghum boiled water extract@20L ha ${ }^{-1}(25$ DAS) & $44.2 \mathrm{ab}$ & $11.0 \mathrm{bcd}(15 \%)$ & $4.8 \mathrm{ab}(25 \%)$ & $90.0 \mathrm{bcd}(2.9 \%)$ & $255.6 \mathrm{a}$ \\
\hline Sorghum boiledwater extract@25Lha-1 $(25$ DAS $)$ & $43.5 \mathrm{abc}$ & $11.8 \mathrm{ab}(9.1 \%)$ & 4.6 abc $(23 \%)$ & $92.5 \mathrm{bc}(5.5 \%)$ & $263.2 \mathrm{a}$ \\
\hline Sorghum boiledwater extract@30L ha-1 $(25$ DAS) & 38.5 bcde & 10.1 ef $(8.0 \%)$ & $4.0 \mathrm{cdef}(12 \%)$ & $86.7 \mathrm{~d}(.72 \%)$ & $227.4 \mathrm{bc}$ \\
\hline Sorghumpowder@270gha-1 (25 DAS) & $43.7 \mathrm{abc}$ & $11.6 \mathrm{abc}(20 \%)$ & $4.7 \mathrm{ab}(25 \%)$ & 88.2 bcd $(.99 \%)$ & $264.4 \mathrm{a}$ \\
\hline Sorghum powder@360g ha ${ }^{-1}(25$ DAS $)$ & $41.2 \mathrm{abcd}$ & $11.5 \mathrm{abc}(21 \%)$ & $4.7 \mathrm{ab}(24 \%)$ & $91.0 \mathrm{bcd}(3.9 \%)$ & $258.3 \mathrm{a}$ \\
\hline Sorghum powder@450gha-1 (25DAS) & $36.7 \mathrm{de}$ & 10.4 de $(14 \%)$ & 4.2 bcde $(16 \%)$ & $87.5 \mathrm{~cd}(.14 \%)$ & $230.3 \mathrm{~b}$ \\
\hline Sorghumpowder@540g ha ${ }^{-1}(25$ DAS) & $41.0 \mathrm{abcd}$ & $10.3 \mathrm{de}(13 \%)$ & $4.4 \operatorname{abcd}(20 \%)$ & 90.0 bcd $(2.9 \%)$ & $256.0 \mathrm{a}$ \\
\hline LSD Value $\mathrm{p}<0.05$ & 6.6 & 0.90 & 0.59 & 5.5 & 16.8 \\
\hline \multicolumn{6}{|c|}{ Means sharing an letter in common does not differ significantly at $\leq 5 \%$ probability level } \\
\hline \multicolumn{6}{|c|}{ DAS stands for days after sowing: Lha $^{-1}$ represents liters per hectare } \\
\hline
\end{tabular}

The net benefits of sorghum water boiled extract at the application of 15, 20, 25 and 30 liters per hectare ranged from 872 \$ to 1056 US \$. Whereas, sorghum powder applied amounts in 270, 360, 450 and $540 \mathrm{~g} \mathrm{ha}^{-1}$ had benefits at value of 933 \$ to 1044 \$. Applied sorghum water extract at a volume of $330 \mathrm{~L} \mathrm{ha}^{-1}$ bestow the benefits of value $1015 \$$. On the other hand, to the extent of marginal benefits (MRR\%), the herbicide application had an expenditure of $16.69 \$$, but remained still economical with $315 \%$ MRR. Sorghum extract as soaked water extract application at a volume of 330 liters on per hectare basis gave the marginal rate of return of $154.55 \$$, whereas sorghum water boiled extraction at a volume of 25 liters per hectare gave an MRR of 5000\%. Application of water (second control) was also economical (1371\% MRR). Whereas, the remaining treatments were found dominated owing to high associated costs and less value of benefits, thus can be considered uneconomical. 
Muhammad Waqas Aslam Cheema et al.

Table-5: Economic analysis of the treatments

\begin{tabular}{|c|c|c|c|c|c|c|c|c|c|c|c|c|c|}
\hline Parameters & $\mathbf{T}_{1}$ & $\mathbf{T}_{2}$ & $\mathbf{T}_{3}$ & $\mathbf{T}_{4}$ & $\mathbf{T}_{5}$ & $T_{6}$ & $\mathbf{T}_{7}$ & $\mathbf{T}_{8}$ & $\mathbf{T}_{9}$ & $T_{10}$ & $T_{11}$ & $\mathbf{T}_{12}$ & Remarks \\
\hline Grain yield & 3.5 & 3.8 & 5.0 & 4.6 & 3.9 & 4.8 & 4.6 & 4.0 & 4.7 & 4.7 & 4.2 & 4.4 & \\
\hline $\begin{array}{c}\text { Adjusted } \\
\text { yield }\end{array}$ & 3.2 & 3.4 & 4.5 & 4.1 & 3.5 & 4.3 & 4.2 & 3.6 & 4.2 & 4.2 & 3.8 & 4.0 & $\begin{array}{c}\mathrm{t} \mathrm{ha}^{-1} \text { (10\% reduction to bring } \\
\text { at farmers level) }\end{array}$ \\
\hline $\begin{array}{c}\text { Gross } \\
\text { income }\end{array}$ & 82915 & 88065 & 117677 & 106836 & 92004 & 111240 & 108227 & 94502 & 110081 & 109386 & 98493 & 104055 & $\begin{array}{c}\text { Wheat price at }=\text { PKR } 25750 \\
\text { tonn }\end{array}$ \\
\hline $\begin{array}{c}\text { Cost of } \\
\text { herbicide }\end{array}$ & 0 & 0 & 1750 & 0 & 0 & 0 & 0 & 0 & 0 & 0 & 0 & 0 & $\begin{array}{c}\text { Herbicide iodosulforun } \\
\text { +mesosulforun@14.4 g ha }{ }^{-1} \\
\text { (Atlantis @ 3.6 WG a.i)= } \\
\text { Rs.750/160g }\end{array}$ \\
\hline $\begin{array}{l}\text { Cost of } \\
\text { extracts } \\
\text { and } \\
\text { powder } \\
\text { formation }\end{array}$ & 0 & 0 & 0 & 115 & 150 & 200 & 250 & 300 & 217.5 & 290 & 362.5 & 435 & $\begin{array}{l}\text { Sorghum herbage }=40 \\
\text { PKR } / 40 \mathrm{~kg} \text { as } 1 \mathrm{PKR} / 1 \mathrm{~kg} . \\
\text { Labour cost for soaking } \\
\text { sorghum }=75 \mathrm{PKR} \\
\text { Expenditure on preparation of } \\
\text { sorghum water boiled extracts } \\
=(\mathrm{Rs} 10 / \mathrm{L})(150 / 15 \mathrm{~L} \\
\text { Expenditure on sorghum } \\
\text { allelopathic powder Via spray } \\
\text { dryer }=250 \text { Rs per kg }\end{array}$ \\
\hline $\begin{array}{c}\text { Sprayer } \\
\text { rent }\end{array}$ & 0 & 50 & 50 & 50 & 50 & 50 & 50 & 50 & 50 & 50 & 50 & 50 & PKR 50 spray $^{-1}$ \\
\hline \begin{tabular}{|c|} 
Spray \\
application \\
cost \\
\end{tabular} & 0 & 300 & 300 & 300 & 300 & 300 & 300 & 300 & 300 & 300 & 300 & 300 & $\begin{array}{c}\text { PKR } 300 \text { man }^{-1} \text { day }^{-1} \text { (one } \\
\text { man-day ha }{ }^{-1} \text { ) }\end{array}$ \\
\hline $\begin{array}{l}\text { Total cost } \\
\text { that vary }\end{array}$ & 0 & 350 & 2100 & 465 & 500 & 550 & 600 & 650 & 567.5 & 640 & 712.5 & 785 & PKR \\
\hline $\begin{array}{c}\text { Net } \\
\text { benefits }\end{array}$ & 82915 & 87715 & 115577 & 106363 & 91475 & 110690 & 107627 & 93852 & 109513 & 108746 & 97780 & 103270 & PKR ha ${ }^{-1}$ \\
\hline \multicolumn{14}{|c|}{ 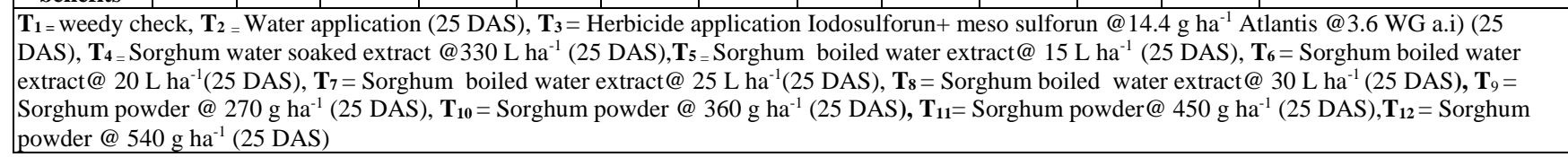 } \\
\hline
\end{tabular}

Table-6: Marginal rates of returns from the experiment

\begin{tabular}{|c|c|c|c|c|c|}
\hline Treatments & $\begin{array}{l}\text { Cost that } \\
\text { varies }\end{array}$ & Net profits & Marginal cost & $\begin{array}{c}\text { Marginal } \\
\text { net benefits }\end{array}$ & MRR\% \\
\hline Weedy check & 0 & 82915 & - & - & - \\
\hline Water application (25 DAS) & 350 & 87715 & 350 & 4800 & 1371 \\
\hline Sorghum soaked water extract@ $330 \mathrm{~L} \mathrm{ha}^{-1}$ (25 DAS) & 465 & 106363 & 115 & 18648 & 16200 \\
\hline Sorghum boiled water extract@15 L ha $a^{-1}$ (25 DAS) & 500 & 91475 & $\mathrm{D}$ & $\mathrm{D}$ & $\mathrm{D}$ \\
\hline Sorghum boiled water extract@ $20 \mathrm{~L} \mathrm{ha}^{-1}$ (25 DAS) & 550 & 110690 & 85 & 4327 & 5000 \\
\hline Sorghum powder @270 $\mathrm{g} \mathrm{ha}^{-1}(25 \mathrm{DAS})$ & 567.5 & 109513 & $\mathrm{D}$ & $\mathrm{D}$ & $\mathrm{D}$ \\
\hline Sorghum boiled water extract@ 25 L ha ${ }^{-1}$ (25 DAS) & 600 & 107627 & $\mathrm{D}$ & $\mathrm{D}$ & $\mathrm{D}$ \\
\hline Sorghum powder @ $360 \mathrm{~g} \mathrm{ha}^{-1}$ (25 DAS) & 640 & 108746 & $\mathrm{D}$ & $\mathrm{D}$ & $\mathrm{D}$ \\
\hline Sorghum powder@ $30 \mathrm{~L} \mathrm{ha}^{-1}$ (25 DAS) & 650 & 93852 & $\mathrm{D}$ & $\mathrm{D}$ & $\mathrm{D}$ \\
\hline Sorghum powder @ $450 \mathrm{~g} \mathrm{ha}^{-1}$ (25 DAS) & 712 & 97780 & $\mathrm{D}$ & $\mathrm{D}$ & $\mathrm{D}$ \\
\hline Sorghum powder @ $540 \mathrm{~g} \mathrm{ha}^{-1}(25 \mathrm{DAS})$ & 785 & 103270 & $\mathrm{D}$ & $\mathrm{D}$ & $\mathrm{D}$ \\
\hline $\begin{array}{l}\text { Herbicide application iodosulforun+ meso sulforun } \\
@ 14.4 \mathrm{~g} \mathrm{ha}^{-1} \text { Atlantis @3.6 WG a.i)(@ 25 DAS) }\end{array}$ & 2100 & 115577 & 1550 & 4887 & 315 \\
\hline
\end{tabular}




\section{Discussion}

The phenomena of allelochemicals have been explored in many ways like crop rotation, cover crops, mulching, water extracts, powder formulations, intercropping and residues application (Farooq et al., 2013; Jabran et al., 2015). But here, this experiment was conducted for field appraisal of sorghum in various ways. As, the inhibitory role of allelopathy has been well documented by many researchers (Cheng and Cheng, 2015; Jabran et al., 2015; Glab et al., 2017), It was observed considerable difference among the treatments applied in the form of soaked and boiled water formulations and in the powder form in comparison with the herbicide. The reduction in the weed density observed in this study is also due to this dimension of allelochemicals produced by the sorghum plant when get assorted with the water and its allelopathic interaction. Significant reductions in weed density were described by Cheema and Khaliq (2000) in sorghum allelopathic evaluation. The outcomes are in agreement with the conclusion of Jamil et al. (2009) whom pragmatic a decline in Phalaris minor dry weight by $36-55 \%$ by sorghum + sunflower water extract at $12 \mathrm{~L} \mathrm{ha}^{-1}$.

It was observed that the sorghum water boiled extract, sorghum powder and sorghum soaked water extract drastically reduced weeds DW, as presented in the Table (3). The effect up to various extents is due to variable contact surface made by these formulations. Therefore, a treatment would be more effective in effect, if it covers more surface area weed plant. From some previous related studies, it was found that conclusion of present experiment nearly compatible with Cheema and Khaliq, (2000) and Jamil et al. (2009) whom made use of sorghum water extract for potential allelopathic crops. They have reported a reduction in weeds dry weight as well. The reductions in dry biomass of Phalaris minor were 21-58\%, whereas, in the case of Chenopodium album the reduction was in between $38-60 \%$.

All these results can be followed and for these findings the justification lies in various possible mechanisms revealed about sorghum allelopathic potential. The allelochemicals can interfere with the hormonal and enzymatic activities. Therefore, it may affect directly or indirectly the processes like nutrient uptake and transport within the plant, stomatal oscillations, cell membrane permeability, and the most important, the photosynthesis and respiration processes (Cheng and Cheng, 2015). The economic and marginal evaluation of the treatments were conducted according to the method prescribed by Byerlee (1988). Farmers are more concerned about profits and acceptability of a practice or technology mainly dependent upon on the value of earnings. Here, estimations were made to review the gains in each treatment and on every increase in cost that diverge. As far as the economics is concerned, higher benefits were observed by herbicide application. Significant rise in yield was owing to weed suppression and it had higher benefits that are well convincing for the farmers. Therefore, it can state that rise in yield was owing to substantial weed control that enhanced the competency potential of crop to exploit the maximum available possessions (Jabran et al., 2015).

\section{Conclusion}

It is concluded that allelopathy can be a useful technique to manage the weeds in wheat and foliar applied water-soaked sorghum extract at a volume of $330 \mathrm{~L} \mathrm{ha}^{-1}$ or as boiled water extract at $20 \mathrm{~L} \mathrm{ha}^{-1}$ can be an economically feasible way for controlling the weeds. It can be recommended as a sustainable approach, while sorghum powder preparation by evaporation process from the above extracts can be too expensive procedure for the farmers.

\section{Acknowledgement}

The authors are cordially thankful to Prof. (Rtd.) Dr. Zahid Atta Cheema and Dr. Muhammad Farooq, for their guidance to complete the present study. All the work was carried out by facilities provided at UAF under the projects of Higher Education Commission Pakistan, Pakistan Science Foundation and Pakistan Agricultural Research Council. 
Disclaimer: None.

Conflict of Interest: None.

Source of Funding: The study was funded through research projects of Higher Education Commission (HEC) of Pakistan, Pakistan Science Foundation and Pakistan Agricultural Research Council.

\section{References}

Arif M, Cheema ZA, Khaliq A and Hassan A, 2015. Organic weed management in wheat through allelopathy. Int. J. Agric. Biol. 17: 127-134.

Anne LG, Lum JB, Dayan FE, Locke ME, Sejero SH and Jacobsen CS, 2009. Mineralization of the allelochemicals sorgoleone in soil. Chemosphere. 76: 1041-1047.

Bilalis D, Papastylianou P, Konstantas A, Patsiali S, Karkanis A and Efthimiadou A, 2010. Weedsuppressive effects of maize-legume intercropping in organic farming. Int. J. Pest Manage. 56: 173-181.

Byerlee D, 1988. From agronomic data to farmer recommendation: An economics training manual. CIMMYT, Mexico, D.F., pp. 31-33.

Cheema ZA and Khaliq A, 2000. Use of sorghum allelopathic properties to control weeds in irrigated wheat in a semi-arid region of Punjab. Agriculture, Ecosyst. Environ. 79: 105-112.

Cheema ZA, Khaliq A and Saeed S, 2004. Weed control in maize (Zea mays L.) through sorghum allelopathy. J. Sustain. Agric. 23(4): 73-86.

Corley JC, 2015. Pest management: trends and challenges. Int. J. Pest Manage. 61: 1-2.

Cheng F and Cheng Z, 2015. Research progress on the use of plant allelopathy in agriculture and the physiological and ecological mechanisms of allelopathy. Front. Plant Sci. 6: 1020.

Farooq M, Bajwa AA, Cheema SA and Cheema ZA, 2013. Application of allelopathy in crop production. Int. J. Agric. Biol. 15: 1367-1378.

Farooq M, Jabran K, Cheema ZA, Wahid A and Siddique KHM, 2011. The role of allelopathy in agricultural pest management. Pest Manage. Sci. 67: 494-506.

Glab L, Sowinski J, Bough R and Dayan FE, 2017. Allelopathic potential of sorghum (Sorghum bicolor (L.) Moench) in weed control: A comprehensive review. Adv. Agron. 145: 43-95.

Iqbal MM, Murtaza G, Naz T Javed W, Hussain S, Ilyas M, Anjum MA, Shahzad SM, Ashraf M and Iqbal $\mathrm{Z}, 2017$. Uptake, translocation of $\mathrm{Pb}$ and chlorophyll contents of Oryza sativa as influenced by soil applied amendments under normal and salt-affected $\mathrm{Pb}$-spiked soil conditions. Asian J. Agric. Biol. 5(1):15-25.

Jamil M, Cheema ZA, Mushtaq MN, Farooq M and Cheema MA, 2009. Alternative control of wild oat and canary grass in wheat fields by allelopathic plant water extracts. Agron. Sustain. Develop. 2: 475-482.

Jabran K, Mahajan G, Sardana V and Chauhan BS, 2015. Allelopathy for weed control in agricultural systems. Crop Prot. 72: 57-65.

Kadioglu I and Farooq S, 2017. Potential distribution of sterile oat (Avena sterilis L.) in Turkey under changing climate. Turkish J. Weed Sci. 20(2): 113.

Khan M and Damlas CA, 2015. Factors preventing the adoption of alternatives to chemical pest control among Pakistani cotton farmers. Int. J. Pest Manage. 61: 9-16.

Large EC, 1954. Growth stages in cereals illustration of the Feekes Scale. Plant Pathol. 3(4): 128-129.

Mahmood A and Cheema ZA, 2004. Influence of sorghum mulch on purple nutsedge (Cyperus rotundus L.). Int. J. Agric. Biol. 6(1): 86-88.

Mazid M, Khan TA and Mohammad F, 2011. Role of secondary metabolites in defense mechanisms of plants. Biol. Med. 3(2): 232-249.

Silva RS, Tomaz AC, Lopes MC, Martins JC, Xavier VM and Picanco MC, 2015. Toxicity of botanical insecticides on Diaphania hyalinata, their selectivity for the predatory ant Paratrechina sp. and their potential phytoxicty on pumpkin. Int. J. Pest Manage. 62: 95-104.

Smith J, Whereley B, Reynolds C, White R, Senseman S and Falk S, 2015. Weed control spectrum and turfgrass tolerance to bioherbicide Phoma macrostoma. Int. J. Pest Manage. 61(2): 91-98.

Torun H and Uygur S, 2012. Damages caused by different doses of herbicides application on some crops. Turkish J. Weed Sci. 19(2): 28-37. 
Weston LA, Alsaadawi IS and Baerson SR, 2013. Sorghum allelopathy-from ecosystem to molecule. J. Chem. Ecol. 39: 142-153.

Zhu Y and Li QX, 2002. Movement of bromacil and hexazinone in soils of Hawaiian pineapple fields. Chemosphere. 49: 669-674.

Zohaib A, Ehsanullah, Tabassum T, Abbas T and Rasool T, 2014. Influence of water soluble phenolics of Vicia sativa L. on germination and seedling growth of pulse crops. Sci. Agric. 8: 148151.

\section{Contribution of Authors}

Cheema MWA: Conducted Research, analyzed the data and interpreted the results

Rasool T: Helped to shape the research article, data evaluation and manuscript handling

Munir H: Conceived the idea of experiment and hypothesis formulation and implementation of treatments

Iqbal MM: Provided critical feedback in manuscript handling, proof reading and contributed to the final version of the manuscript

Naz T: Proof read for technical details in the manuscript

Haq MIU: Helped in performing the field experimental activities

Mustafa A: Helped in planning of experiment, analytical work and data collection

Nadeem M: Contributed in collection and analysis of data

Ullah S: Assisted in sample collection, handling and yield estimations 